\title{
Fc gamma receptor llla polymorphisms in advanced colorectal cancer patients correlated with response to anti-EGFR antibodies and clinical outcome
}

Rosa Calemma ${ }^{1 \dagger}$, Alessandro Ottaiano ${ }^{2 \dagger}$, Anna Maria Trotta', Guglielmo Nasti ${ }^{2}$, Carmela Romano ${ }^{2}$, Maria Napolitano ${ }^{1}$, Domenico Galati ${ }^{1}$, Pasquale Borrelli ${ }^{1}$, Serena Zanotta ${ }^{1}$, Antonino Cassata ${ }^{2}$, Giuseppe Castello ${ }^{3}$, Vincenzo Rosario laffaioli ${ }^{2}$ and Stefania Scala ${ }^{1 *}$

\begin{abstract}
Background: Anti-EGFR monoclonal antibodies have shown efficacy in the treatment of metastatic colorectal cancer (mCRC). One of the mechanism is the antibody-dependent cell-mediated cytotoxicity (ADCC) in which Fc region of the antibody binds to the Fc gamma receptors (FcyR) expressed by immune cells. The present study investigated the association between single nucleotide polymorphisms of FcyRlla and FcyRIlla and clinical outcome in $\mathrm{mCRC}$ treated with anti-EGFR antibodies.

Methods: Seventy-four consecutive patients with mCRC were analyzed. The genotypes for FcyRlla-131 histidine $(H)$ /arginine (R), FcyRllla-158 valine (V)/phenylanaline (F) polymorphisms were evaluated by directly sequencing. Multiplex allele-specific polymerase chain reaction was performed for FcyRllla-158 valine (V)/phenylanaline (F). Correlations between FcyR polymorphisms, baseline patient and tumor features were studied by contingency tables and the chi-square test. The Kaplan-Meier product limit method was applied to the progression-free survival (PFS) curves. Univariate analysis was performed with the log-rank test. Cox proportional-hazards regression was used to analyze the effect of multiple risk factors on PFS.

Results: FcyRllla polymorphisms were significantly associated with response to anti-EGFR-based therapy in 49 patients with kras wt tumors ( $p=0.035$ ). There was not association with response for FcyRlla polymorphisms. Furthermore, obtained results suggested that prognosis is particularly unfavorable for patients carrying the FcyRllla-158F/F genotype (median PFS VN, V/F, F/F: 18.2 vs 17.3 vs 9.4 months). No prognostic ability was identified for FcyRlla polymorphisms.
\end{abstract}

Conclusions: In mCRC patients the presence of FcyRIlla-F can predict resistance to anti-EGFR therapy and unfavorable prognosis.

Keywords: Fc gamma receptor, Colorectal cancer, Prognosis, Cetuximab, Panitumumab, Antibody-dependent cell-mediated cytotoxicity

\footnotetext{
* Correspondence: s.scala@istitutotumori.na.it

${ }^{\dagger}$ Equal contributors

'Oncological Immunology, National Cancer Institute "G. Pascale",

via M. Semmola, Naples 80131, Italy

Full list of author information is available at the end of the article
} 


\section{Background}

Metastatic colorectal cancer (mCRC) is the second most common cause of cancer death in the Western world accounting for $40-50 \%$ of newly diagnosed patients [1]. Despite therapeutic advances, the prognosis for patients with $\mathrm{mCRC}$ remains poor. However, the addition of drugs such as irinotecan and oxaliplatin to 5-fluorouracil (5-FU) has almost doubled the median survival from 12 months to 21 months [2]. Monoclonal antibodies (mAbs) binding to the vascular endothelial growth factor (VEGF) (bevacizumab) or the epidermal growth factor receptor (EGFR) (cetuximab and panitumumab) have shown efficacy in the treatment of mCRC increasing the life expectancy of patients by more than 2 years [3]. While bevacizumab is administered in combination with chemotherapy as a first-line treatment, anti-EGFR mAbs find place in later-line treatments.

Cetuximab is an IgG1a chimeric mAb while panitumumab is a fully human IgG2 mAb; they bind to EGFR and block the binding of its natural ligands, preventing ligand dependent homodimerization and activation of intracellular cascades that control cellular proliferation, adhesion, angiogenesis, and apoptosis. Anti-EGFR mAbs have proven to be effective in combination with chemotherapy or as single agents for treatment of mCRC [3]. Recent evidences showed that $\mathrm{mCRC}$ responds differently to EGFR-targeted agents on genetic basis that involve also the EGFR downstream effectors (i.e. kras, braf, PIK3CA and PTEN) [4]. Although largely unexplored, monoclonal antibodies also induce antibody-dependent cell-mediated cytotoxicity (ADCC) [5-8]. ADCC is induced through the interaction of the $\mathrm{Fc}_{\mathrm{c}}$ region of the $\mathrm{mAb}$ with the $\mathrm{Fc}_{\mathrm{C}}$ gamma receptor $(\mathrm{Fc} \gamma \mathrm{R})$ expressed by effector cells (i.e. natural killer-NK-lymphocytes, monocytes/macrophages). Polymorphisms have been demonstrated on genes encoding for the activating receptors FcyRIIa (CD32, mainly expressed on macrophages) and FcyRIIIa (CD16, expressed on NK cells and macrophages) [9], affecting their affinity to human IgG: a histidine $(\mathrm{H})$ /arginine $(\mathrm{R})$ polymorphism at position 131 for FcyRIIa and a valine (V)/phenylalanine (F) polymorphism at position 158 for FcyRIIIa. Based on the different affinities, patients harboring Fc $\gamma$ RIIa- $131 \mathrm{H} / \mathrm{H}$ and FcyRIIIa-4 158V/V genotypes would be expected to mediate a more efficient ADCC antitumor response. Clinical studies utilizing rituximab in the treatment of B-cell nonHodgkin's lymphoma have shown that FcyRIIa-131H/H and FcyRIIIa-158V/V genotypes were associated with better clinical outcome [10,11]. Patients with 158V/V and/or $131 \mathrm{H} / \mathrm{H}$ had a significantly higher response rate than patients without either genotype (59\% vs $18 \%$ ). The progression-free survival (PFS) estimate of patients with $158 \mathrm{~V} / \mathrm{V}$ and/or $131 \mathrm{H} / \mathrm{H}$ allele was also significantly longer, with median PFS of 445 and 140 days for the two groups, respectively [11]. Nevertheless it was shown that when
CT is added to Rituximab the predictive value of FCGR polymorphisms was lost probably due to the high efficacy of CT [12]. In trastuzumab-treated metastatic breast cancer, ADCC analysis showed that the combination of $158 \mathrm{~V} / \mathrm{V}$ and/or $131 \mathrm{H} / \mathrm{H}$ had a significantly higher trastuzumab-mediated cytotoxicity than other genotypes in addition to higher response rate and a longer PFS [13]. Contrasting results have been reported on the role of Fc $\gamma \mathrm{R}$ polymorphisms in mCRC $[14,15]$. Recently, it was described that FcyRIIa-131H/H and FcyRIIIa-158F/F polymorphisms associated with better PFS in a series of EGFR-expressing mCRC patients treated with single-agent cetuximab [14]. Conversely, Bibeau et al. demonstrated a favourable effect on PFS only for the FcyRIIIA-158V/V genotype unrelated to the kras status [15]. The goal of our study was to explore the association between FcyRIIa and FcyRIIIa polymorphisms and the outcome of mCRC patients treated with anti-EGFR-based therapies (cetuximab and panitumumab).

\section{Methods \\ Patient management and follow-up}

Seventy-four stage IV CRC patients were studied at the Division of Abdominal Medical Oncology of the National Cancer Institute (Naples, Italy) from May 2007 to May 2009. Patients were eligible after specific discussion on the study. Informed consent from each patient was sought. The protocol was conducted according to a protocol approved by the institutional review board/independent ethics committee. Patients were routinely characterized for kras mutational status [16]. All patients underwent to sequential standard treatments based on chemotherapy and/or biologic therapies (bevacizumab, cetuximab, panitumumab). First and second-line chemotherapy (CT) included the association of fluoropyrimidines (capecitabine or 5-fluoruracile) with oxaliplatin or irinotecan. The chemotherapy regimen was based on patient's performance status, extent of disease, comorbidities, previous treatments and individual preferences. Some selected patients underwent pulmonary and/or liver metastasectomies as established in a multidisciplinary team discussion. Bone metastases were treated with palliative radiotherapy. Patients features are shown in Table 1. Cetuximab or panitumumab were administered only in patients with kras wilde-type (wt) tumors. All patients underwent first-line chemotherapy, 54 patients (72.9\%) received a second-line chemotherapy and 22 (29.7\%) a third-line chemotherapy. Eight patients received palliative radiotherapy. Eleven patients with advanced disease underwent to palliative resection of primary colonic tumor. Metastasectomies before or after chemotherapy were performed in 26 patients. Total body computed tomography scan and CEA monitoring were done every three 
Table 1 Detailed characteristics of patients and tumors

\begin{tabular}{|c|c|c|c|c|c|c|c|c|c|c|c|c|c|c|}
\hline $\begin{array}{l}\text { Patient } \\
\text { Initials }\end{array}$ & Gender & $\begin{array}{c}\text { Age } \\
\text { (years) }\end{array}$ & $\begin{array}{c}\text { Mucinous } \\
\text { component }>50 \%\end{array}$ & $\begin{array}{l}\text { Primary } \\
\text { tumor }\end{array}$ & Grading & $\begin{array}{l}\text { Stage at } \\
\text { diagnosis }\end{array}$ & pT & FcgRIIIA & FcgRIIA & $\begin{array}{c}\text { PFS } \\
\text { (months) }\end{array}$ & $\begin{array}{l}\text { First-line } \\
\text { CT }\end{array}$ & $\begin{array}{l}\text { Response to } \\
\text { first-line CT }\end{array}$ & $\begin{array}{l}\text { Anti-EGFR } \\
\text { therapy }\end{array}$ & $\begin{array}{c}\text { Response to } \\
\text { anti-EGFR therapy }\end{array}$ \\
\hline $\mathrm{IN}$ & Female & 57 & Yes & Colon & 3 & 4 & 3 & $158 \mathrm{~V} N(\mathrm{G} / \mathrm{G})$ & $131 \mathrm{H} / \mathrm{H}(\mathrm{A} / \mathrm{A})$ & 20,0 & $\mathrm{FU}+\mid \mathrm{RI}+\mathrm{BEV}$ & PR & CET & $P R$ \\
\hline $\mathrm{MM}$ & Female & 50 & No & Rectum & 3 & 4 & NA & $158 \mathrm{~V} / \mathrm{F}(\mathrm{G} / \mathrm{T})$ & $131 \mathrm{H} / \mathrm{R}(\mathrm{A} / \mathrm{G})$ & 12,1 & $\mathrm{CAPE}+\mathrm{OXA}+\mathrm{BEV}$ & NA & CET & SD \\
\hline SU & Male & 38 & No & Rectum & 2 & 3 & 2 & $158 \mathrm{~V} N(\mathrm{G} / \mathrm{G})$ & $131 \mathrm{H} / \mathrm{R}(\mathrm{A} / \mathrm{G})$ & 17,9 & $\mathrm{FU}+\mid \mathrm{RI}+\mathrm{BEV}$ & PR & CET & $P R$ \\
\hline RAM & Female & 70 & Yes & Rectum & 2 & 1 & 2 & 158V/F (G/T) & $131 \mathrm{H} / \mathrm{H}(\mathrm{A} / \mathrm{A})$ & 10,2 & $\mathrm{FU}+\mathrm{OXA}+\mathrm{BEV}$ & $C R$ & CET & SD \\
\hline AA & Female & 77 & No & Colon & 3 & 4 & 3 & $158 \mathrm{~V} / \mathrm{F}(\mathrm{G} / \mathrm{T})$ & $131 \mathrm{H} / \mathrm{H}(\mathrm{A} / \mathrm{A})$ & 6,9 & $\mathrm{FU}+\mathrm{OXA}+\mathrm{BEV}$ & SD & CET & SD \\
\hline $\mathrm{MC}$ & Male & 67 & No & Rectum & 3 & 4 & 4 & $158 \mathrm{~V} N(\mathrm{G} / \mathrm{G})$ & $131 \mathrm{H} / \mathrm{R}(\mathrm{A} / \mathrm{G})$ & 6,6 & $\mathrm{CAPE}+\mathrm{OXA}+\mathrm{BEV}$ & PD & CET & SD \\
\hline $\mathrm{MU}$ & Male & 82 & NA & Colon & 2 & 3 & 3 & $158 \mathrm{~V} N(\mathrm{G} / \mathrm{G})$ & $131 \mathrm{H} / \mathrm{R}(\mathrm{A} / \mathrm{G})$ & NP & $\mathrm{CAPE}+\mathrm{OXA}+\mathrm{BEV}$ & SD & No & NA \\
\hline AN & Female & 76 & No & Colon & 2 & 4 & NA & $158 \mathrm{~V} N(\mathrm{G} / \mathrm{G})$ & $131 \mathrm{H} / \mathrm{H}(\mathrm{A} / \mathrm{A})$ & NP & $\mathrm{CAPE}+\mathrm{OXA}+\mathrm{BEV}$ & $P R$ & No & NA \\
\hline VA & Female & 60 & No & Colon & 3 & 4 & NA & 158V/F(G/T) & $131 \mathrm{H} / \mathrm{R}(\mathrm{A} / \mathrm{G})$ & 12,1 & $\mathrm{CAPE}+\mathrm{OXA}+\mathrm{BEV}$ & $\mathrm{PR}$ & No & NA \\
\hline GA & Female & 70 & No & Colon & 3 & 4 & 4 & $158 \mathrm{~V} N(\mathrm{G} / \mathrm{G})$ & $131 \mathrm{H} / \mathrm{R}(\mathrm{A} / \mathrm{G})$ & 18,2 & CAPE+OXA & $\mathrm{PR}$ & CET & SD \\
\hline$S A$ & Female & 62 & NA & Rectum & 3 & 4 & 3 & 158V/F (G/T) & $131 \mathrm{H} / \mathrm{R}(\mathrm{A} / \mathrm{G})$ & 6,4 & $\mathrm{FU}+\mid \mathrm{RI}+\mathrm{BEV}$ & PD & CET & PD \\
\hline $\mathrm{PG}$ & Male & 75 & No & Colon & 3 & 1 & 2 & 158F/F (T/T) & $131 \mathrm{H} / \mathrm{R}(\mathrm{A} / \mathrm{G})$ & 14,8 & $\mathrm{FU}+\mathrm{OXA}$ & $\mathrm{PR}$ & PAN & $P R$ \\
\hline$\overline{L R B}$ & Male & 61 & No & Colon & 1 & 3 & 3 & 158F/F (T/T) & $131 \mathrm{H} / \mathrm{R}(\mathrm{A} / \mathrm{G})$ & 7,7 & $\mathrm{FU}+|\mathrm{R}|+\mathrm{BEV}$ & SD & CET & SD \\
\hline$\overline{V A}$ & Male & 71 & No & Colon & 2 & 4 & NA & $158 \mathrm{~V} / \mathrm{G} / \mathrm{G})$ & $131 \mathrm{H} / \mathrm{R}(\mathrm{A} / \mathrm{G})$ & 8,8 & $\mathrm{CAPE}+\mathrm{OXA}+\mathrm{BEV}$ & PD & No & NA \\
\hline$S G$ & Male & 55 & No & Colon & 2 & 4 & NA & $158 \mathrm{~V} / \mathrm{G}(\mathrm{G} / \mathrm{G})$ & $131 \mathrm{H} / \mathrm{H}(\mathrm{A} / \mathrm{A})$ & 19,7 & $\mathrm{FU}+\mid \mathrm{RI}+\mathrm{BEV}$ & PD & No & NA \\
\hline $\mathrm{MR}$ & Female & 61 & No & Colon & 3 & 4 & NA & $158 \mathrm{~V} / \mathrm{G}(\mathrm{G} / \mathrm{G})$ & $131 \mathrm{H} / \mathrm{R}(\mathrm{A} / \mathrm{G})$ & 10,8 & $\mathrm{CAPE}+\mathrm{OXA}+\mathrm{BEV}$ & SD & No & NA \\
\hline MMG & Female & 43 & No & Colon & 2 & 3 & 4 & $158 \mathrm{~V} N(\mathrm{G} / \mathrm{G})$ & $131 \mathrm{H} / \mathrm{H}(\mathrm{A} / \mathrm{A})$ & 16,1 & $\mathrm{FU}+\mathrm{OXA}+\mathrm{BEV}$ & $P R$ & PAN & $P R$ \\
\hline$\overline{M M}$ & Male & 74 & No & Colon & 2 & 4 & 3 & 158V/F (G/T) & $131 \mathrm{H} / \mathrm{H}(\mathrm{A} / \mathrm{A})$ & NP & $\mathrm{FU}+|\mathrm{R}|+\mathrm{BEV}$ & SD & No & NA \\
\hline$\overline{G M}$ & Male & 72 & No & Rectum & 3 & 4 & NA & 158F/F (T/T) & $131 \mathrm{H} / \mathrm{R}(\mathrm{A} / \mathrm{G})$ & 2,6 & CAPE+BEV & SD & PAN & NA \\
\hline LCR & Male & 74 & No & Rectum & 3 & 3 & 3 & $158 \mathrm{~V} / \mathrm{G}(\mathrm{G} / \mathrm{G})$ & $131 \mathrm{H} / \mathrm{R}(\mathrm{A} / \mathrm{G})$ & 23,0 & CAPE & $C R$ & CET & $P R$ \\
\hline$B G$ & Female & 47 & No & Colon & 3 & 4 & NA & 158F/F (G/T) & $131 \mathrm{H} / \mathrm{R}(\mathrm{A} / \mathrm{G})$ & NP & $\mathrm{CAPE}+\mathrm{OXA}+\mathrm{BEV}$ & $C R$ & No & NA \\
\hline LG & Female & 56 & No & Colon & 3 & 3 & 3 & $158 \mathrm{~V} / \mathrm{G} / \mathrm{G})$ & $131 \mathrm{H} / \mathrm{R}(\mathrm{A} / \mathrm{G})$ & 22,4 & $\mathrm{FU}+\mid \mathrm{RI}+\mathrm{BEV}$ & PD & CET & SD \\
\hline$\overline{\mathrm{DMV}}$ & Male & 65 & No & Colon & 2 & 3 & 3 & 158V/F (G/T) & $131 \mathrm{H} / \mathrm{R}(\mathrm{A} / \mathrm{G})$ & 22,8 & $\mathrm{CAPE}+\mathrm{OXA}+\mathrm{BEV}$ & $\mathrm{PR}$ & CET & $P R$ \\
\hline FA & Female & 66 & No & Colon & 2 & 4 & 4 & 158V/F (G/T) & $131 \mathrm{H} / \mathrm{H}(\mathrm{A} / \mathrm{A})$ & 10,0 & $\mathrm{FU}+\mid \mathrm{RI}+\mathrm{BEV}$ & PR & CET & $P R$ \\
\hline $\mathrm{GF}$ & Male & 56 & No & Colon & 3 & 4 & NA & 158V/F (G/T) & $131 \mathrm{H} / \mathrm{H}(\mathrm{A} / \mathrm{A})$ & $\mathrm{NP}$ & $\mathrm{FU}+\mid \mathrm{RI}+\mathrm{BEV}$ & $\mathrm{PR}$ & No & NA \\
\hline MA & Female & 55 & No & Rectum & 2 & 4 & NA & 158V/F (G/T) & $131 \mathrm{H} / \mathrm{H}(\mathrm{A} / \mathrm{A})$ & NP & CAPE+OXA+BEV & $\mathrm{PR}$ & No & NA \\
\hline MMR & Female & 54 & No & Rectum & 2 & 1 & 2 & 158V/F (G/T) & $131 \mathrm{H} / \mathrm{H}(\mathrm{A} / \mathrm{A})$ & NA & $\mathrm{FU}+\mid \mathrm{RI}+\mathrm{BEV}$ & SD & No & NA \\
\hline LS & Female & 58 & No & Colon & 3 & 1 & NA & 158V/F (G/T) & $131 \mathrm{H} / \mathrm{R}(\mathrm{A} / \mathrm{G})$ & 15,6 & $\mathrm{FU}+|\mathrm{R}|+\mathrm{BEV}$ & PR & CET & PR \\
\hline$C D$ & Male & 64 & No & Rectum & 3 & 4 & NA & 158V/F (G/T) & 131H/R (A/G) & 7,0 & FU+|R|+BEV & CR & CET & $P R$ \\
\hline $\mathrm{VE}$ & Female & 56 & No & Colon & 2 & 3 & 3 & 158V/F (G/T) & $131 \mathrm{H} / \mathrm{H}(\mathrm{A} / \mathrm{A})$ & 38,0 & $\mathrm{FU}+\mid \mathrm{RI}+\mathrm{BEV}$ & PR & CET & $P R$ \\
\hline VA & Male & 81 & No & Colon & 2 & 4 & NA & $158 \mathrm{~V} N(\mathrm{G} / \mathrm{G})$ & $131 \mathrm{H} / \mathrm{R}(\mathrm{A} / \mathrm{G})$ & 8,9 & $\mathrm{FU}+|\mathrm{R}|+\mathrm{BEV}$ & PR & CET & SD \\
\hline $\mathrm{CM}$ & Female & 75 & No & Colon & 2 & 2 & 3 & 158V/F (G/T) & $131 \mathrm{H} / \mathrm{H}(\mathrm{A} / \mathrm{A})$ & 9,8 & $\mathrm{CAPE}+\mathrm{OXA}+\mathrm{BEV}$ & SD & PAN & $P R$ \\
\hline$\overline{I G}$ & Male & 73 & No & Colon & 3 & 3 & NA & 158V/F (G/T) & $131 \mathrm{H} / \mathrm{H}(\mathrm{A} / \mathrm{A})$ & 19,5 & $\mathrm{FU}+|\mathrm{R}|+\mathrm{BEV}$ & SD & CET & $C R$ \\
\hline$\overline{\mathrm{DGS}}$ & Male & 63 & No & Rectum & 1 & 4 & NA & $158 \mathrm{~V} N(\mathrm{G} / \mathrm{G})$ & $131 \mathrm{H} / \mathrm{R}(\mathrm{A} / \mathrm{G})$ & 7,7 & CAPE+OXA & $C R$ & CET & $\mathrm{PD}$ \\
\hline
\end{tabular}


Table 1 Detailed characteristics of patients and tumors (Continued)

\begin{tabular}{|c|c|c|c|c|c|c|c|c|c|c|c|c|c|c|}
\hline $\mathrm{RO}$ & Female & 47 & No & Rectum & 2 & 3 & 4 & 158V/F (G/T) & $131 R / R(G / G)$ & 9,2 & CAPE+OXA & PR & CET & SD \\
\hline VA & Male & 79 & No & Colon & 2 & 4 & NA & $158 \mathrm{~V} N(\mathrm{G} / \mathrm{G})$ & $131 \mathrm{H} / \mathrm{R}(\mathrm{A} / \mathrm{G})$ & 13,3 & $\mathrm{FU}+|\mathrm{R}|+\mathrm{BEV}$ & PR & CET & SD \\
\hline DFC & Male & 64 & NA & Colon & 2 & 4 & 3 & 158V/F $(\mathrm{G} / \mathrm{T})$ & $131 \mathrm{H} / \mathrm{R}(\mathrm{A} / \mathrm{G})$ & 21,3 & CAPE+BEV & PR & CET & SD \\
\hline$\overline{E l}$ & Female & 64 & No & Colon & 2 & 4 & NA & $158 \mathrm{~V} / \mathrm{F}(\mathrm{G} / \mathrm{T})$ & $131 \mathrm{H} / \mathrm{H}(\mathrm{A} / \mathrm{A})$ & 16,2 & $\mathrm{CAPE}+\mathrm{OXA}+\mathrm{BEV}$ & $P R$ & No & NA \\
\hline DAR & Female & 66 & Yes & Colon & 2 & 4 & NA & 158V/F(G/T) & $131 \mathrm{H} / \mathrm{H}(\mathrm{A} / \mathrm{A})$ & $\mathrm{NP}$ & $\mathrm{CAPE}+\mathrm{OXA}+\mathrm{BEV}$ & $C R$ & No & NA \\
\hline BT & Male & 56 & No & Rectum & 2 & 4 & NA & $158 \mathrm{~V} / \mathrm{G} / \mathrm{G})$ & $131 \mathrm{H} / \mathrm{R}(\mathrm{A} / \mathrm{G})$ & NP & $\mathrm{FU}+|\mathrm{R}|+\mathrm{BEV}$ & PR & No & NA \\
\hline CAG & Female & 54 & No & Rectum & 2 & 3 & 4 & $158 \mathrm{~V} N(\mathrm{G} / \mathrm{G})$ & $131 \mathrm{H} / \mathrm{H}(\mathrm{A} / \mathrm{A})$ & 11,7 & $\mathrm{CAPE}+|\mathrm{R}|+\mathrm{BEV}$ & $\mathrm{PR}$ & CET & $\mathrm{PR}$ \\
\hline DV & Female & 78 & No & Colon & 3 & 3 & 3 & 158V/F (G/T) & $131 \mathrm{H} / \mathrm{R}(\mathrm{A} / \mathrm{G})$ & 43,6 & $\mathrm{FU}+|\mathrm{R}|+\mathrm{BEV}$ & PR & CET & $P R$ \\
\hline $\mathrm{BG}$ & Male & 65 & No & Colon & 3 & 4 & NA & 158V/F (G/T) & $131 \mathrm{H} / \mathrm{H}(\mathrm{A} / \mathrm{A})$ & 18,3 & $\mathrm{FU}+|\mathrm{R}|+\mathrm{BEV}$ & SD & CET & $P R$ \\
\hline NG & Male & 80 & No & Colon & 2 & 4 & NA & 158F/F (T/T) & $131 \mathrm{H} / \mathrm{H}(\mathrm{A} / \mathrm{A})$ & 5,4 & FU & PD & No & NA \\
\hline NR & Female & 42 & No & Colon & 3 & 4 & NA & 158V/F(G/T) & $131 \mathrm{H} / \mathrm{R}(\mathrm{A} / \mathrm{G})$ & 6,7 & $\mathrm{CAPE}+\mathrm{OXA}+\mathrm{BEV}$ & PD & No & NA \\
\hline $\mathrm{MR}$ & Male & 60 & No & Colon & 3 & 4 & 3 & 158V/F (G/T) & 131H/R (A/G) & 8,7 & $\mathrm{FU}+|\mathrm{R}|+\mathrm{BEV}$ & $\mathrm{PR}$ & CET & SD \\
\hline SF & Female & 62 & No & Rectum & 2 & 4 & NA & $158 \mathrm{~V} / \mathrm{F}(\mathrm{G} / \mathrm{T})$ & 131R/R(G/G) & 14,2 & CAPE & SD & CET & SD \\
\hline DCM & Male & 60 & No & Colon & 2 & 4 & NA & 158V/F (G/T) & $131 \mathrm{H} / \mathrm{R}(\mathrm{A} / \mathrm{G})$ & 37,5 & $\mathrm{FU}+|\mathrm{R}|+\mathrm{BEV}$ & $P R$ & CET & $P R$ \\
\hline CML & Female & 44 & No & Colon & 3 & 3 & 3 & 158V/F (G/T) & $131 \mathrm{H} / \mathrm{R}(\mathrm{A} / \mathrm{G})$ & 29,3 & $\mathrm{CAPE}+\mathrm{OXA}+\mathrm{BEV}$ & $C R$ & CET & PR \\
\hline $\mathrm{BM}$ & Male & 66 & No & Rectum & 3 & 4 & NA & $158 \mathrm{~V} N(\mathrm{G} / \mathrm{G})$ & $131 \mathrm{H} / \mathrm{R}(\mathrm{A} / \mathrm{G})$ & 8,1 & CAPE+BEV & SD & CET & SD \\
\hline $\mathrm{Ml}$ & Female & 40 & NA & Colon & 2 & 4 & NA & $158 \mathrm{~V} / \mathrm{G} / \mathrm{G})$ & $131 \mathrm{H} / \mathrm{R}(\mathrm{A} / \mathrm{G})$ & NP & FU+|RI+BEV & PR & No & NA \\
\hline$\overline{G C}$ & Female & 57 & No & Rectum & 2 & 4 & NA & $158 \mathrm{~V} / \mathrm{G} / \mathrm{G})$ & $131 \mathrm{H} / \mathrm{H}(\mathrm{A} / \mathrm{A})$ & NP & $\mathrm{CAPE}+\mathrm{OXA}+\mathrm{BEV}$ & PR & No & NA \\
\hline SC & Male & 53 & No & Rectum & 3 & 4 & NA & $158 \mathrm{~V} / \mathrm{G} / \mathrm{G})$ & $131 \mathrm{H} / \mathrm{R}(\mathrm{A} / \mathrm{G})$ & NP & $\mathrm{CAPE}+\mathrm{OXA}+\mathrm{BEV}$ & SD & No & NA \\
\hline $\mathrm{RR}$ & Male & 77 & No & Colon & 2 & 2 & 1 & 158V/F (G/T) & $131 \mathrm{H} / \mathrm{R}(\mathrm{A} / \mathrm{G})$ & 9,4 & $\mathrm{FU}+\mathrm{OXA}+\mathrm{BEV}$ & PR & PAN & SD \\
\hline $\mathrm{GA}$ & Female & 70 & No & Colon & 3 & 4 & 4 & $158 \mathrm{~V} N(\mathrm{G} / \mathrm{G})$ & $131 \mathrm{H} / \mathrm{R}(\mathrm{A} / \mathrm{G})$ & 14,6 & CAPE+OXA & $\mathrm{PR}$ & No & SD \\
\hline $\mathrm{MP}$ & Male & 78 & No & Colon & 3 & 4 & 3 & 158V/F (G/T) & $131 \mathrm{H} / \mathrm{R}(\mathrm{A} / \mathrm{G})$ & 9,6 & $\mathrm{CAPE}+\mathrm{OXA}+\mathrm{CET}$ & SD & CET & SD \\
\hline$C M$ & Male & 67 & No & Rectum & 3 & 3 & 3 & $158 \mathrm{VN}(\mathrm{G} / \mathrm{G})$ & $131 \mathrm{H} / \mathrm{H}(\mathrm{A} / \mathrm{A})$ & 43,6 & $|\mathrm{R}|+\mathrm{CET}$ & $C R$ & CET & SD \\
\hline FR & Female & 55 & Yes & Rectum & 3 & 4 & NA & $158 \mathrm{~V} / \mathrm{G} / \mathrm{G})$ & 131R/R(G/G) & 12,4 & CAPE+OXA & SD & CET & SD \\
\hline $\mathrm{GL}$ & Male & 74 & Yes & Colon & 2 & 4 & NA & $158 \mathrm{~V} N(\mathrm{G} / \mathrm{G})$ & $131 \mathrm{H} / \mathrm{H}(\mathrm{A} / \mathrm{A})$ & NP & $\mathrm{FU}+\mathrm{OXA}+\mathrm{BEV}$ & PR & No & NA \\
\hline TMA & Female & 66 & No & Colon & 1 & 4 & NA & $158 \mathrm{~V} N(\mathrm{G} / \mathrm{G})$ & $131 \mathrm{H} / \mathrm{R}(\mathrm{A} / \mathrm{G})$ & NP & $\mathrm{FU}+\mid \mathrm{RI}+\mathrm{BEV}$ & $\mathrm{PR}$ & No & NA \\
\hline CF & Female & 71 & NA & Rectum & 2 & 4 & NA & $158 \mathrm{~V} N(\mathrm{G} / \mathrm{G})$ & $131 \mathrm{H} / \mathrm{R}(\mathrm{A} / \mathrm{G})$ & NP & CAPE+|R|+BEV & $\mathrm{PR}$ & No & NA \\
\hline$\overline{C E}$ & Female & 55 & No & Colon & 1 & 1 & 4 & $158 \mathrm{VN}(\mathrm{G} / \mathrm{G})$ & $131 R / R(G / G)$ & 6,1 & CAPE+|R|+CET & SD & CET & SD \\
\hline SME & Male & 63 & No & Colon & 3 & 4 & 4 & $158 \mathrm{VN}(\mathrm{G} / \mathrm{G})$ & $131 \mathrm{H} / \mathrm{R}(\mathrm{A} / \mathrm{G})$ & NP & $C A P E+|R|+C E T$ & PR & CET & PR \\
\hline GM & Male & 56 & No & Rectum & 3 & 4 & 4 & 158F/F (T/T) & $131 \mathrm{H} / \mathrm{H}(\mathrm{A} / \mathrm{A})$ & 7,2 & $\mathrm{CAPE}+\mathrm{OXA}+\mathrm{CET}$ & PR & CET & PD \\
\hline $\mathrm{IP}$ & Male & 69 & No & Rectum & 3 & 4 & NA & $158 \mathrm{~V} / \mathrm{G} / \mathrm{G})$ & $131 \mathrm{H} / \mathrm{H}(\mathrm{A} / \mathrm{A})$ & 7,8 & CAPE+OXA & $\mathrm{PR}$ & CET & PR \\
\hline TMR & Female & 68 & Yes & Colon & 3 & 4 & NA & $158 \mathrm{~V} N(\mathrm{G} / \mathrm{G})$ & $131 \mathrm{H} / \mathrm{R}(\mathrm{A} / \mathrm{G})$ & 8,8 & CAPE+OXA+CET & PD & CET & SD \\
\hline$\overline{P M}$ & Female & 77 & No & Colon & 1 & 3 & 3 & 158F/F (T/T) & $131 \mathrm{H} / \mathrm{H}(\mathrm{A} / \mathrm{A})$ & 9,4 & CAPE+OXA & $C R$ & CET & PD \\
\hline$M G$ & Male & 64 & No & Rectum & 3 & 4 & NA & 158V/F (G/T) & $131 \mathrm{H} / \mathrm{H}(\mathrm{A} / \mathrm{A})$ & 16,5 & $\mid \mathrm{RI}+\mathrm{CET}$ & PD & CET & SD \\
\hline$\overline{M G}$ & Male & 68 & No & Colon & 3 & 4 & NA & $158 \mathrm{~V} / \mathrm{F}(\mathrm{G} / \mathrm{T})$ & $131 \mathrm{H} / \mathrm{H}(\mathrm{A} / \mathrm{A})$ & 2,8 & CAPE+OXA & PD & CET & PD \\
\hline PG & Male & 64 & No & Rectum & 3 & 2 & 3 & $158 \mathrm{~V} / \mathrm{F}(\mathrm{G} / \mathrm{T})$ & $131 \mathrm{H} / \mathrm{H}(\mathrm{A} / \mathrm{A})$ & 37 & FU & PR & CET & SD \\
\hline
\end{tabular}


Table 1 Detailed characteristics of patients and tumors (Continued)

\begin{tabular}{|c|c|c|c|c|c|c|c|c|c|c|c|c|c|c|}
\hline SS & Male & 82 & No & Rectum & 3 & 4 & NA & 158V/F (G/T) & $131 \mathrm{H} / \mathrm{R}(\mathrm{A} / \mathrm{G})$ & 19,4 & CAPE & $\mathrm{PR}$ & CET & PD \\
\hline FP & Male & 69 & Yes & Colon & 3 & 4 & NA & 158V/F (G/T) & $131 \mathrm{H} / \mathrm{R}(\mathrm{A} / \mathrm{G})$ & 20,8 & $\mathrm{FU}+\mid \mathrm{RI}$ & SD & CET & PR \\
\hline $\mathrm{PA}$ & Female & 70 & NA & Colon & 2 & 4 & 3 & $158 \mathrm{~V} / \mathrm{F}(\mathrm{G} / \mathrm{T})$ & $131 \mathrm{H} / \mathrm{H}(\mathrm{A} / \mathrm{A})$ & 23,3 & CAPE+OXA & $C R$ & No & NA \\
\hline DLA & Male & 82 & No & Colon & 3 & 3 & 2 & $158 \mathrm{~F} / \mathrm{F}(\mathrm{T} / \mathrm{T})$ & $131 \mathrm{H} / \mathrm{R}(\mathrm{A} / \mathrm{G})$ & 28,0 & $\mathrm{CAPE}+\mathrm{OXA}$ & SD & CET & PD \\
\hline
\end{tabular}

CT: Chemotherapy: NA: Not Assessable; NP: Not Progressed; CR: Complete Response; PR: Partial Response; SD: Stable Disease; PD: Progressive Disease; PFS: Progression Free Survival; FU: Fluorouracile; IRI: Irinotecan; CAPE: Capecitabine; BEV: Bevacizumab; PAN: Panitumumab; CET: Cetuximab. 
months. The response to therapy was evaluated by RECIST criteria. Patients with target metastatic lesions restaged at the Radiology Unit were considered for response evaluation.

Complete response (CR) was defined as complete disappearance of all detectable evidence of disease on total body computed tomography. Partial response (PR) was defined as at least a $30 \%$ decrease in the sum of diameters of target lesions. Stable disease (SD) was defined as everything between $30 \%$ decrease and $20 \%$ growth of tumor size. Progressive disease (PD) was defined as at least a $20 \%$ increase in the sum of diameters of target lesions. Two patients were lost at follow-up.

Analysis of FcyRIla-H131R, FcyRIIla-V158F polymorphisms Genomic DNA was extracted from white blood cells (WBCs) using a DNA extraction kit.(Qiagen,Valencia, $\mathrm{CA}$ ) and stored at $-20^{\circ}$. FcyRIIa genotyping was performed on genomic DNA by polymerase chain reaction
(PCR) method adapted from a previously established protocol [17]. Briefly, PCR amplification was performed in $50 \mu \mathrm{l}$ reaction mixture containing $100 \mathrm{ng}$ genomic DNA, $0.4 \mathrm{mM}$ of each primer, $0.4 \mathrm{mM}$ dNTPs, 20 mM Tris- $\mathrm{HCl}, \mathrm{pH}$ 9.0, $100 \mathrm{mM} \mathrm{KCl,} 0.1 \mathrm{mM}$ EDTA, 1.0 mM DTT, $0.5 \%$ tween 20 , and $1 \mathrm{U}$ Taq DNA polymerase. The program was performed in the thermal Cycler 2770 by Applied Biosystems and consisted of an initial denaturation step at $95^{\circ} \mathrm{C}$ for $5^{\prime}$, followed by 36 cycles of $95^{\circ} \mathrm{C}$ for 30 seconds, $56^{\circ} \mathrm{C}$ for 40 seconds, $72^{\circ} \mathrm{C}$ for 40 seconds and a final elongation step at $72^{\circ} \mathrm{C}$ for $10 \mathrm{~min}$ utes. The primers used for PCR amplification were forward primer $5^{\prime}$-GGAGAAACCATCATGCTGAG-3' and reverse primer 5'-CAATTTTGCTGCTATGGGC-3'. The resulting PCR product (289bp) was purified with the Montage $\mathrm{SEQ}_{96}$ Sequencing Reaction Cleanup Kits (Millipore) and prepared to sequence through a second PCR reaction using Big Dye Terminator v3.1 Cycle Sequencing Kit by Applied Biosystems in forward and reverse direction

a

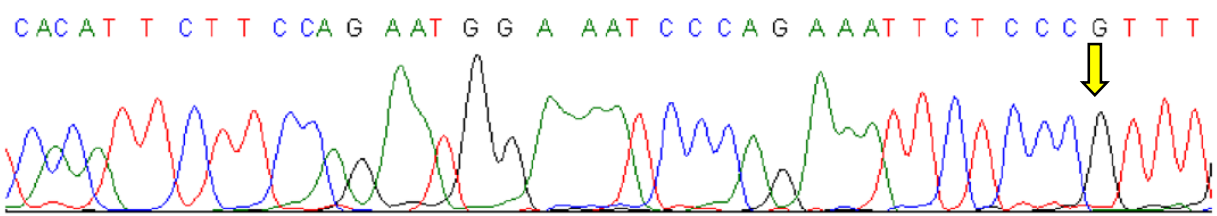

b
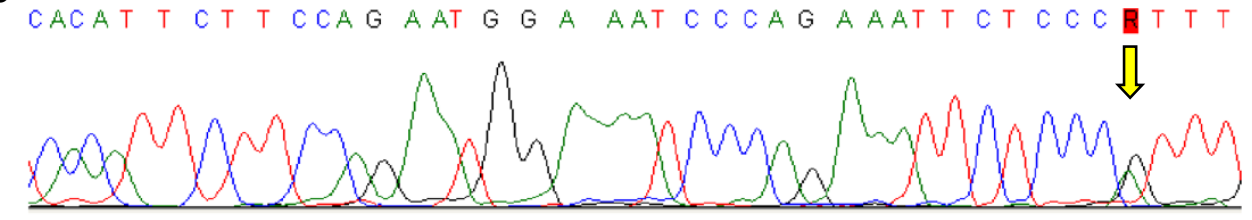

C
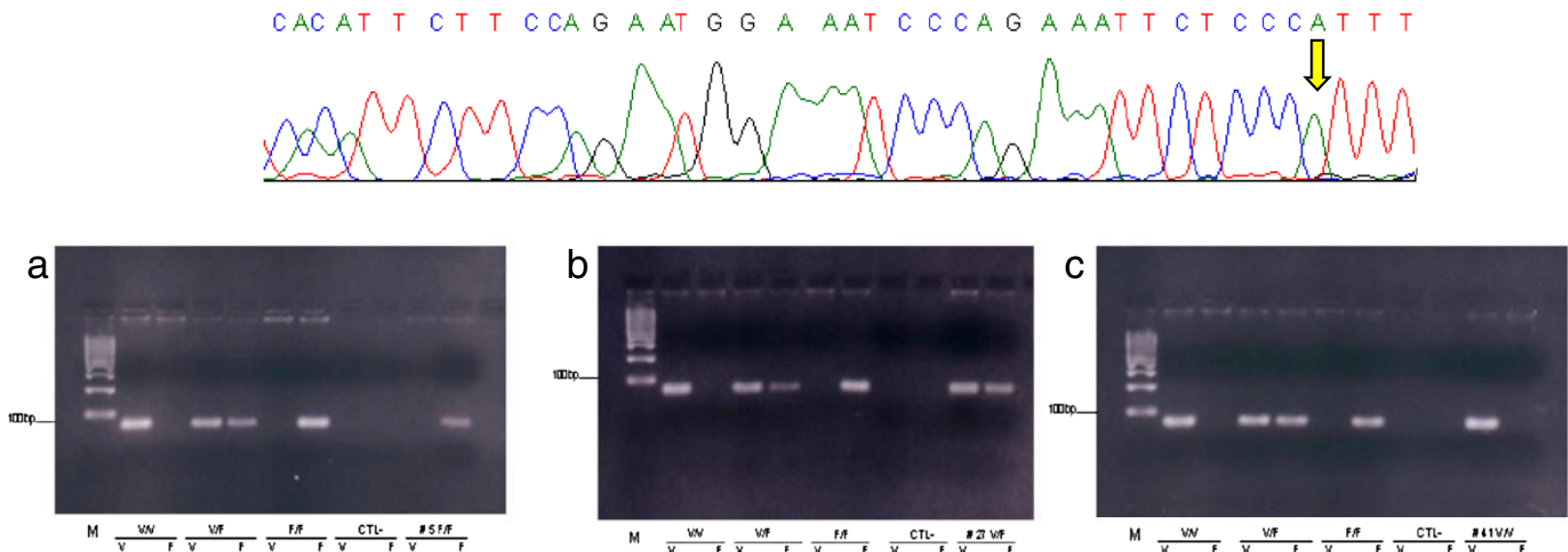

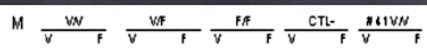

Figure 1 FcyRlla determined by direct sequencing and FcyRIIla allotyping by allele-specific PCR. $\mathbf{v}$ Upper Panel. FcyRlla determined by direct sequencing: (a) Sequencing electropherogram obtained from a sample homozygous for allele FcyRlla 131H/H; (b) Sequencing electropherogram obtained from a sample heterozygous for allele FcyRlla 131H/R; (c) Sequencing electropherogram obtained from a sample homozygous for allele FcyRIla 131R/R. Lower Panel. FcyRIlla allotyping by allele-specific PCR. 100bp ladder marker, FcyRllla genotypes direct sequenced F/F, V/F and $V / V$ control and CTL- negative control were represented. Examples represented respectively F/F (a), V/F (b) and VN (c) patients. 
of the region of interest $\left(96^{\circ} \mathrm{C}\right.$ for 1 minutes, 25 cycle of $96^{\circ} \mathrm{C}$ for 10 seconds, $56^{\circ} \mathrm{C}$ for 5 seconds and $60^{\circ} \mathrm{C}$ for 2 minutes). PCR product was purified with Montage SEQ 96 Sequencing Reaction Cleanup Kits (Millipore) and direct sequencing was run with Applied Biosystems3130 Genetic Analyzers (Figure 1).

For FcyRIIIa-V158F polymorphism, allele-specific PCR method was followed. Briefly, 100 ng of genomic DNA was amplified using allele-specific common forward primer 5'TCCAAAAGCCACACTCAAAGAC-3' and reverse primer 5'-CTGAAGACACATTTTTACTCCCAAAC-3'. PCR amplification was performed in $25 \mu$ l reaction mixture containing $100 \mathrm{ng}$ genomic DNA, $0.3 \mathrm{mM}$ of each primer, $0.2 \mathrm{mM}$ dNTPs, $20 \mathrm{mM}$ Tris- $\mathrm{HCl}, \mathrm{pH}$ 9.0, $100 \mathrm{mM} \mathrm{KCl}$, $0.1 \mathrm{mM}$ EDTA, $1.0 \mathrm{mM}$ DTT, $0.5 \%$ tween 20 , and $1 \mathrm{U}$ Taq DNA polymerase. The program was performed in the thermal Cycler 2770 by Applied Biosystems and consisted of an initial denaturation step at $95^{\circ} \mathrm{C}$ for $5^{\prime}$, followed by 35 cycles of $94^{\circ} \mathrm{C}$ for 30 seconds, $64^{\circ} \mathrm{C}$ for 30 seconds, $72^{\circ} \mathrm{C}$ for 30 seconds and a final elongation step at $72^{\circ} \mathrm{C}$ for 10 minutes. Three DNA samples previously sequenced FcgRIIIa-V/V158, FcgRIIIa-V/F158, FcgRIIIa-F/F158 were run in all reactions (Figure 1). The reaction products were run on $3 \%$ ethidium bromide-stained agarose gel. Seventy-three base pair PCR fragment either positive for valine $(\mathrm{V})$ or $\mathrm{F}$ allele was visualized under UV light as reported previously [18]. To confirm FcyRIIIa genotype automatic sequencing was performed using forward primer 5' - TGT AAA ACG ACG GCC AGT TCA TCA TAA TTC TGT CTT CT-3'; reverse primer 5'-CAG GAA ACA GCT ATG ACC CTT GAG TGA TGG TGA TGT TCA-3'. The part of exon 4 which contains the polymorphic site was amplified by PCR using $100 \mathrm{ng}$ genomic DNA, $0.4 \mathrm{mM}$ of each primer, $0.4 \mathrm{mM}$ dNTPs, 20 mM Tris- $\mathrm{HCl}, \mathrm{pH}$ 9.0, $100 \mathrm{mM} \mathrm{KCl} \mathrm{,} 0.1$ mM EDTA, 1.0 mM DTT, $0.5 \%$ tween 20, and $1 \mathrm{U}$ Taq DNA polymerase. The program was performed in the thermal Cycler 2770 by Applied Biosystems and consisted of an initial denaturation step at $95^{\circ} \mathrm{C}$ for $5^{\prime}$, followed by 36 cycles of $95^{\circ} \mathrm{C}$ for 30 seconds, $57^{\circ} \mathrm{C}$ for 30 seconds, $72^{\circ} \mathrm{C}$ for 30 seconds and a final elongation step at $72^{\circ} \mathrm{C}$ for $10 \mathrm{~min}$ utes. The PCR product was sequenced using the Big Dye
Terminator v3.1 Cycle Sequencing Kit by Applied Biosystems.

\section{Statistical analyses and data presentation}

Associations between $\mathrm{F} c \gamma \mathrm{R}$ polymorphisms and clinical pathologic variables were evaluated by $x^{2}$ test. $\mathrm{p}<0.05$ was considered statistically significant. Genotype data for FcyR polymorphisms and clinicpathological variables were retrospectively collected and associated with response to anti-EGFR-based therapy by $X^{2}$ test with level of significance set at $\mathrm{p}<0.05$. Progression-free survival (PFS) was defined as the time elapsed from the treatment initiation and tumor progression or death from any cause. The Kaplan-Meier product limit method was applied to graph PFS. Univariate analysis was done with the log-rank test. Cox proportional hazards regression was used to analyze the effect of several risk factors on PFS. Risk factors (covariates) were: age, sex, grading, response to $\mathrm{I}^{\circ}$ line chemotherapy, $\mathrm{F}_{\mathrm{c}} \mathrm{\gamma}$ p polymorphisms. Ninety-five percent confidence intervals of hazard ratios were also reported. No attempts were done to analyze overall survival because of low events. Seventy-two patients were analyzed since two were lost at follow-up. Statistical analysis was performed using the MedCalc ${ }^{\circledR}$ 9.3.7.0 and Excel software.

\section{Results}

\section{Characteristics of patients and tumors}

Seventy-four patients seen from May 2007 to May 2009 were studied for the FcyRIIa and FcyRIIIa polymorphisms through direct sequencing and allele specific PCR as reported in Figure 1. Patients features are detailed in Table 1. Median age was 65 years. Genders were equally represented. Twenty-six tumors originated in the rectum $51.4 \%$ of patients had highgrade (G3) disease. The majority of lesions presented with a pT3 extent of invasion at diagnosis and 22 presented with $\mathrm{pN}+$ disease. Fifty patients presented with stage IV disease, 16 with stage III and 8 with stage I/II. The majority of tumors (90.5\%) did not

Table 2 Response to anti-EGFR therapy according to FcyR polymorphisms

\begin{tabular}{|c|c|c|c|c|c|c|c|}
\hline & \multirow[b]{2}{*}{ Total no.(\%) } & \multicolumn{3}{|c|}{ FcyRIIla } & \multicolumn{3}{|c|}{ FcyRlla } \\
\hline & & $\mathrm{V} / \mathrm{V}$ & $\mathrm{V} / \mathrm{F}$ & $\overline{F / F}$ & $\mathrm{H} / \mathrm{H}$ & $H / R$ & $R / R$ \\
\hline \multicolumn{8}{|c|}{$\begin{array}{l}\text { Response to anti-EGFR based chemotherapy } \\
\text { (49 KRAS-wt evaluable pts) }\end{array}$} \\
\hline $\mathrm{CR}+\mathrm{PR}$ & $20(40.8)$ & 7 & 12 & 1 & 9 & 11 & 0 \\
\hline$\overline{S D}$ & $22(44.9)$ & 10 & 11 & 1 & 5 & 13 & 4 \\
\hline$\overline{\mathrm{PD}}$ & $7(14.3)$ & 1 & 3 & 3 & 3 & 4 & 0 \\
\hline $\bar{P}$ & & 0.035 & & & 0.344 & & \\
\hline
\end{tabular}


Table 3 Correlation between skin toxicity and response to anti-EGFR therapy (A), and polymorphisms

\begin{tabular}{lccccc}
\hline & \multicolumn{4}{c}{ Response to anti-EGFR therapy } & \\
\cline { 2 - 5 } & CR & PR & SD & PD & $P^{* *}$ \\
\hline Skin toxicity grade* & & & & & \\
\hline Grade 1 & 0 & 3 & 7 & 5 & \\
\hline Grade 2 & 0 & 4 & 11 & 2 & \\
\hline Grade 3 & 1 & 12 & 4 & 0 & 0.005 \\
\hline
\end{tabular}

have a mucinous component; the most represented histology was pure colonic adenocarcinoma (Table 1).

FcyRIIla but not FcyRIla polymorphisms were significantly associated with response to anti-EGFR-based therapy in kras wt tumors

Fifty patients were treated with anti-EGFR-based therapy and forty nine were evaluable for clinical response (according to RECIST criteria) and PFS. Forty-five patients were treated with cetuximab, five with panitumumab. The genotypic frequencies of FcgRIIIA and FcgRIIa detected within the analyzed population were $36 \% \mathrm{VV}, 54 \% \mathrm{VF}, 10 \% \mathrm{FF}$ and 36\% HH, 56\% HR, 8\% RR, respectively. The $\chi^{2}$ test showed that there were no significant differences in the genotype frequencies $(\mathrm{p}=0.109$ for V158F; $\mathrm{p}=0.183$ for FcgRIIa) between patients and healthy controls. The genotypic distributions were in Hardy-Weinberg Equilibrium.

Objective responses according to Fc $\gamma \mathrm{R}$ polymorphisms were shown in Table 2. FcyRIIIa but not FcyRIIa polymorphisms were significantly associated with response to anti-EGFR-based therapy in kras wt tumors $(\mathrm{p}=0.035)$. The mean number of anti-EGFR therapy cycles were 15 (range: 5-27) considering panitumumab as single administration every two weeks (one administration $=1$ cycle) and cetuximab weekly (two administrations $=1$ cycle). To evaluate skin toxicity and its predictive role and correlation with FcgR polymorphisms (21), the skin related toxicity was evaluated versus the clinical response. A significant correlation was identified $(\mathrm{p}=0.005)$ between skin toxicity and clinical response (Table 3) while no significant correlation was identified between skin toxicity and the genotype distribution (Table 4).

\section{FcyR polymorphisms predict PFS in $\mathrm{mCRC}$ patients treated with anti-EGFR mAbs}

The anti-EGFR treated patients were analyzed for PFS. As of June 2011, after a median follow-up for alive patients of 22.4 months, 43 patients $(87.7 \%)$ had suffered tumor progression and 19 (44.2\%) had died. Median PFS was 17.0 months. Analysis of prognostic factors for PFS is summarized in Table 5. Grading, response to 1st-line chemotherapy and FcyRIIIa polymorphisms had a significant prognostic value with univariate analysis. No prognostic ability was identified for FcyRIIa polymorphisms. The prognostic value of the grading $(\mathrm{p}=0.04$, HR: $1.83, \mathrm{CI}: 1.01-3.31)$, response to $\mathrm{I}^{\circ}$-line chemotherapy $(\mathrm{p}=0.0004, \mathrm{HR}: 1.86, \mathrm{CI}: 1.32-2.62)$ and Fc $\gamma$ RIIIa $(\mathrm{p}=0.001$, HR:2.35; CI:1.37-4.01) was confirmed with multivariate analysis (Table 5). Hazard ratios of relapse and pattern of Kaplan-Meier estimated curves suggest that prognosis is particularly unfavorable for patients expressing the Fc $\gamma$ RIIIa-158F/F genotype (median PFS V/V, V/F, F/F: 18.2 vs 17.3 vs 9.4 months) (Figure 2).

\section{Discussion}

In this manuscript the value of the FcyRIIa-FcyRIIIa polymorphisms was retrospectively correlated to the efficacy of anti-EGFR therapy in mCRC. FcyRIIIa polymorphisms were significantly associated with response to anti-EGFRbased therapy in 49 valuable patients with kras wt tumors. The results suggested that prognosis is particularly unfavorable for patients expressing the FcyRIIIa-158F/F genotype versus patients carrying a V allele (the FcyRIIIa-158F/V or the FcyRIIIa-158V/V genotypes). On this issue, conflicting results were previously described: Bibeau et al. showed a statistically significant difference in PFS in 69 mCRC patients treated with cetuximab plus irinotecan carrying the the FcyRIIIa-158V/V genotype compared to other combinations expressing an F allele while FcyRIIa polymorphisms did not affect prognosis [15]. Conversely, in a series of 39 EGFR-expressing mCRC patients treated with single-agent cetuximab, Zhang et al. [14] found that FcyRIIa-H131R and FcyRIIIa-V158F polymorphisms were independently associated with better PFS. However, against their hypothesis, Fc $\gamma$ RIIIa-158V/V genotype was associated with more unfavorable clinical outcome. The authors suggest that variants of human IgG1-binding sites

Table 4 polymorphisms

\begin{tabular}{|c|c|c|c|c|c|c|c|c|}
\hline & $\mathrm{V} / \mathrm{V}$ & $\mathrm{V} / \mathrm{F}$ & $\mathrm{F} / \mathrm{F}$ & $P^{* *}$ & $H / H$ & $H / R$ & $\mathrm{R} / \mathrm{R}$ & $P^{* *}$ \\
\hline \multicolumn{9}{|c|}{ Skin toxicity grade* } \\
\hline Grade 1 & 3 & 9 & 3 & & 5 & 10 & 0 & \\
\hline Grade 2 & 7 & 8 & 2 & & 5 & 9 & 3 & \\
\hline Grade 3 & 8 & 9 & 0 & 0.2707 & 7 & 9 & 1 & 0.4198 \\
\hline
\end{tabular}

*According to Common Toxicity Criteria for Adverse Event v3.0 and defined as any grade of rash/acne/dermatitis.

$P^{* *}$ at Chi-Square test. 
Table 5 Uni- and multivariate analyses for progression-free survival (PFS)

\begin{tabular}{|c|c|c|c|c|c|c|}
\hline & Events/Patients & Median PFS(months) & $P^{1}$ & $\mathrm{HR}^{2}$ & $95 \% \mathrm{Cl}^{3}$ & $P^{4}$ \\
\hline \multicolumn{7}{|l|}{ Covariate } \\
\hline Age ( $\leq 70$ vs $>70$ years) & $32 / 36$ vs $11 / 13$ & 17.0 vs 18.0 & 0.50 & 0.61 & $0.30-1.20$ & 0.15 \\
\hline Sex (male vs female) & $24 / 28$ vs $19 / 21$ & 18.3 vs 15.6 & 0.73 & 1.28 & $0.69-2.35$ & 0.42 \\
\hline Grading (G1/G2 vs G3) & $15 / 20$ vs $28 / 29$ & 17.3 vs 13.3 & 0.007 & 1.83 & $1.01-3.31$ & 0.04 \\
\hline Response to $1^{\text {st }}$-line CTO (CR vs PR vs SD vs PD) & $7 / 8$ vs $18 / 23$ vs $12 / 12$ vs $6 / 6$ & 20.1 vs 20.0 vs 9.8 vs 7.6 & 0.0026 & 1.86 & $1.32-2.62$ & 0.0004 \\
\hline FcyRIIla (W vs VF vs FF) & $13 / 18$ vs $25 / 26$ vs $5 / 5$ & 18.2 vs 17.3 vs 9.4 & 0.04 & 2.35 & $1.37-4.01$ & 0.001 \\
\hline FcyRlla (HH vs HR vs RR) & $17 / 18$ vs $23 / 27 v s 3 / 4$ & 16.1 vs 18.2 vs 13.3 & 0.61 & 1.19 & $0.72-1.96$ & 0.49 \\
\hline
\end{tabular}

$P^{1}=\log$ Rank $P$.

$\mathrm{HR}^{2}=\mathrm{Cox}$ regression $\mathrm{HR}$

$\mathrm{Cl}^{3}=$ Confidence Intervals

$P^{4}=$ Cox's Proportional Hazards Regression $P$.

can influence the ADCC by modulating complex interactions with activating (FcyRIIIa) or inhibitory (Fc $\gamma$ RIIb) receptors on effector cells.

In the present study a significant association between FcyRIIIa-158V/V genotype and response to anti-EGFRbased chemotherapy was demonstrated in 49 kras wt patients confirming that the expression of the allele $\mathrm{F}$ predicts a worse response and a shorter PFS. In the evaluated population, 49 patients, $5(10.2 \%)$ carried the FF genotype for FcyRIII and 4 of 49 (8.2\%) the RR genotype for FcyRII. These frequencies differ from those previously reported. In particular, Carlotti et al. investigated on 94 Italian patients affected by follicular lymphoma and thus treated with Rituximab; they reported $30 \mathrm{FF}$ (32\%) and $18 \mathrm{RR}$ (19\%) distribution [12]. Bibeau et al. analyzed a cohort of European subjects affected by mCRC-cetuximab treated, reporting 15 FF (22\%) and 17 (28\%) RR patients. Nevertheless, comparing the allelic frequencies in a population of 168 healthy donors there were no significant differences in the genotype frequencies $\left(x^{2}\right.$ test $\mathrm{p}=0.109$ for V158F; $\mathrm{p}=0.183$ for FcgRIIa). Moreover we can also speculate that, although the studied population represent 74 consecutive mCRC patients coming to our observation, they all showed a good Performance Status (0-1) further validated by the neoadjuvant treatment for 23 patients (Fluopirimidine/ Irinotecan/ Bevacizumab) before hepatic metastasectomy.

The role of ADCC induced by EGFR-specific mAbs may prevent tumor outgrowth or metastasis in vivo, even in cancers insensitive to EGFR signaling inhibition [19]. In fact, evidences accumulated on a complex patients evaluation including the kras status but also EGF/EGFR polymorphisms and downstream pathway mutations. To date, unless a large number of patients have been treated with mAbs there are still two crucial issues: i) a small percent of kras mutated patients respond to cetuximab therapy $[3,4]$ and, ii) although kras wt tumors are potentially sensitive to EGFR-targeted mAbs, not all respond to antiEGFR therapy for multiple target expression, amplification or mutations downstream [4]. Blockade of signal transduction may not be the only mechanism of action
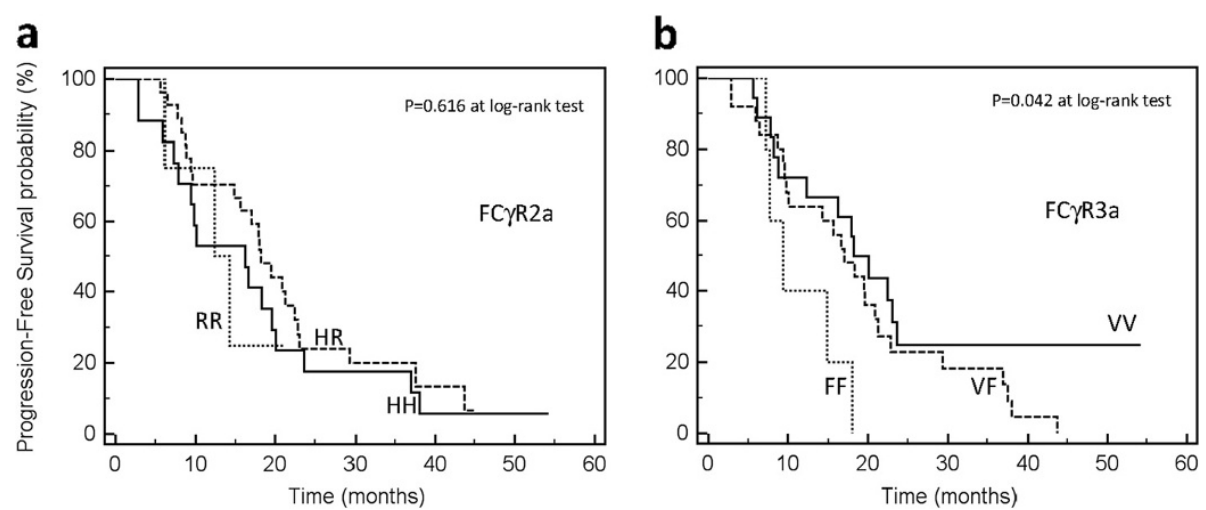

Figure 2 Progression-free survival curves according to FcyR polymorphisms on $\mathbf{4 9} \mathrm{mCR}$ Cpatients. Progression-free survival was defined as time elapsed between treatment initiation and tumor progression (a) FcyRlla: median PFS was 16.1 months in H/H patients (18 patients, 13 events) vs 18.2 months in H/R patients (27 patients, 23 events) vs 13.3 months in R/R patients (4 patients, 3 events); Log Rank test for three curves: $p=0.61$. (b) FcyRllla: median PFS was 18.2 months in VN patients (18 patients, 13 events) vs 17.3 months in V/F patients (26 patients, 25 events) vs 9.4 months in F/F patients ( 5 patients, 5 events); Log Rank test for three curves: $p=0.04$ 
mediating clinical benefit of mAb-treated patients with colorectal cancer [20]. Cell-dependent lysis of target cells mediated by mAbs in vitro and in animal models is a crucial mechanism of action regulated by multiple factors (i.e. Fc $\gamma \mathrm{R}$ on Dendritic Cells, T-helper system, Tregs, B-cells, NK-cells inhibitory proteins, cytokines, etc.). Currently, we are studying the correlation between specific $\mathrm{FC} \gamma \mathrm{R}$ polymorphisms to in vitro ADCC efficacy (Trotta et al., manuscript in preparation).

\section{Conclusions}

Although the exact role of FcyRIIIa-V158F polymorphism and anti-EGFR therapy require more basic studies, the presence of one allele $\mathrm{F}$ of the FcyRIIIa in mCRC patients predicted poor response to anti-EGFR-based therapy and worsen the prognosis.

\section{Competing interests}

The authors declare that they have no competing interests.

\section{Authors' contributions}

$\mathrm{RC}$ and $\mathrm{AO}$ treated the patients and carried out the molecular studies AMT carried out the molecular studies and helped to draft the study. GN participated in its design and coordination and management of patients. CR participated in management of patients. MN carried out the molecular studies and helped to draft the study. DG carried out the molecular studies and helped to draft the study. PB carried out the molecular studies and helped to draft the study. SZ carried out the molecular studies and helped to draft the study. AC participated in its design and coordination and management of patients. GC conceived of the study. VRI conceived of the study, and participated in its design. SS conceived of the study, and participated in its design, statistical analysis and coordination and helped to draft the manuscript. All authors read and approved the final manuscript.

\section{Author details}

'Oncological Immunology, National Cancer Institute "G. Pascale", via M. Semmola, Naples 80131, Italy. ${ }^{2}$ Abdominal Oncology, National Cancer Institute "G. Pascale", via M. Semmola, Naples 80131, Italy. ${ }^{3}$ CROM - Centro Ricerche Oncologiche di Mercogliano, "Fiorentino Lo Vuolo", Via Ammiraglio Bianco, Mercogliano, (AV), Italy.

Received: 5 June 2012 Accepted: 24 October 2012

Published: 21 November 2012

\section{References}

1. Siegel R, Ward E, Brawley O, Jemal A: Cancer statistics, 2011: the impact of eliminating socioeconomic and racial disparities on premature cancer deaths. CA Cancer J Clin 2011, 61:212-236.

2. Davies JM, Goldberg RM: Treatment of metastatic colorectal cancer. Semin Oncol 2011, 38:552-5560.

3. Tol J, Punt CJ: Monoclonal antibodies in the treatment of metastatic colorectal cancer: a review. Clin Ther 2010, 32:437-453.

4. Bardelli A, Siena S: Molecular mechanisms of resistance to cetuximab and panitumumab in colorectal cancer. J Clin Oncol 2010, 28:1254-1261.

5. Ottaiano A, Scala S, laffaioli RV: Cetuximab-dependent ADCC in cancer: dream or reality? Cancer Immunol Immunother 2010, 59:1607-1608.

6. Correale P, Marra M, Remondo C, et al: Cytotoxic drugs up-regulate epidermal growth factor receptor (EGFR) expression in colon cancer cells and enhance their susceptibility to EGFR-targeted antibody-dependent cell-mediated-cytotoxicity (ADCC). Eur J Cancer 2010, 46:1703-1711.

7. Bier H, Hoffmann T, Haas I, van Lierop A: Anti-(epidermal growth factor) receptor monoclonal antibodies for the induction of antibodydependent cell-mediated cytotoxicity 19 against squamous cell carcinoma lines of the head and neck. Cancer Immunol Immunother 1998, 46:167-173.
8. García-Foncillas J, Díaz-Rubio E: Progress in metastatic colorectal cancer: growing role of cetuximab to optimize clinical outcome. Clin Trans/ Oncol 2010, 12:533-542.

9. McKenzie SE, Schreiber AD: Biological advances and clinical applications of Fc receptors for IgG. Curr Opin Hematol 1994, 1:45-52.

10. Cartron G, Dacheux L, Salles $G$, et al: Therapeutic activity of humanized anti-CD2 monoclonal antibody and polymorphism in lgG Fc receptor Fc Rllla gene. Blood 2002, 99:754-758.

11. Weng WK, Levy R: Two immunoglobulin $G$ fragment $C$ receptor polymorphisms independently predict response to rituximab in patients with follicular lymphoma. J Clin Oncol 2003, 21:3940-3947.

12. Carlotti E, Palumbo GA, Oldani E, et al: FcgammaRIIIA and FcgammaRIIIA polymorphisms do not predict clinical outcome of follicular non-Hodgkin's lymphoma patients treated with sequential $\mathrm{CHOP}$ and rituximab. Haematologica 2007, 92:1127-1130

13. Musolino A, Naldi N, Bortesi B, et al: Immunoglobulin G fragment $C$ receptor polymorphisms and clinical efficacy of trastuzumab-based therapy in patients with HER-2/neu-positive metastatic breast cancer. J Clin Oncol 2008, 26:1789-1796.

14. Zhang W, Gordon M, Schultheis AM, et al: Fc R2A and Fc R3A Polymorphisms associated with clinical outcome of epidermal growth factor receptor-expressing metastatic colorectal cancer patients treated with single-agent cetuximab. J Clin Oncol 2007, 25:3712-3718.

15. Bibeau F, Lopez-Crapez E, Di Fiore F, et al: Impact of Fc Rlla-Fc RIlla polymorphisms and KRAS mutations on the clinical outcome of patients with metastatic colorectal cancer treated with cetuximab plus irinotecan J Clin Oncol 2009, 27:1122-1129.

16. Carotenuto P, Roma C, Cozzolino S, et al: Detection of KRAS mutations in colorectal cancer with Fast COLD-PCR. Int J Oncol 2012, 40:378-384.

17. Jiang XM, Arepally G, Poncz M, McKenzie S: Rapid detection of the FcyRIIA-H/R131 ligand-binding polymorphism using an allele-specific restriction enzyme digestion (ASRED). J Immunol Methods 1996, 199:55-59.

18. de Straat FG L-V van der Pol WL, Jansen MD, et al: A novel PCR-based method for direct Fc gamma receptor Illa (CD16) allotyping. J Immunol Methods 2000, 242:127-132.

19. Overdijk MB, Verploegen $S$, van den Brakel JH, et al: Epidermal growth factor receptor (EGFR) antibody-induced antibody-dependent cellular cytotoxicity plays a prominent role in inhibiting tumorigenesis, even of tumor cells insensitive to EGFR signaling inhibition. J Immunol 2011, 187:3383-3390.

20. Van Cutsem E, Tejpar S, Vanbeckevoort D, et al: Intrapatient cetuximab dose escalation in metastatic colorectal cancer according to the grade of early skin reactions: the randomized EVEREST study. J Clin Oncol 2012, 30:2861-2868.

\section{doi:10.1186/1479-5876-10-232}

Cite this article as: Calemma et al:: Fc gamma receptor Illa polymorphisms in advanced colorectal cancer patients correlated with response to anti-EGFR antibodies and clinical outcome. Journal of Translational Medicine 2012 10:232.

\section{Submit your next manuscript to BioMed Central and take full advantage of:}

- Convenient online submission

- Thorough peer review

- No space constraints or color figure charges

- Immediate publication on acceptance

- Inclusion in PubMed, CAS, Scopus and Google Scholar

- Research which is freely available for redistribution 